\title{
Explicit Bounds and Sharp Results for the Composition Operators Preserving the Exponential Class
}

\author{
Fernando Farroni ${ }^{1}$ and Raffaella Giova ${ }^{2}$ \\ ${ }^{1}$ Università Telematica PEGASO, Piazza Trieste e Trento 48, 80132 Napoli, Italy \\ ${ }^{2}$ Dipartimento di Studi Economici e Giuridici, Università degli Studi di Napoli "Parthenope", Palazzo Pacanowsky, \\ Via Generale Parisi 13, 80132 Napoli, Italy
}

Correspondence should be addressed to Raffaella Giova; raffaella.giova@uniparthenope.it

Received 26 April 2016; Accepted 5 July 2016

Academic Editor: Stanislav Hencl

Copyright (C) 2016 F. Farroni and R. Giova. This is an open access article distributed under the Creative Commons Attribution License, which permits unrestricted use, distribution, and reproduction in any medium, provided the original work is properly cited.

\begin{abstract}
Let $f: \Omega \subset \mathbb{R}^{n} \rightarrow \mathbb{R}^{n}$ be a quasiconformal mapping whose Jacobian is denoted by $J_{f}$ and let $\operatorname{EXP}(\Omega)$ be the space of exponentially integrable functions on $\Omega$. We give an explicit bound for the norm of the composition operator $T_{f}: u \in \operatorname{EXP}(\Omega) \mapsto u \circ f^{-1} \in$ $\operatorname{EXP}(f(\Omega))$ and, as a related question, we study the behaviour of the norm of $\log J_{f}$ in the exponential class. The $A_{\infty}$ property of $J_{f}$ is the counterpart in higher dimensions of the area distortion formula due to Astala in the plane and it is the key tool to prove the sharpness of our results.
\end{abstract}

\section{Introduction and Statement of the Main Result}

This paper is concerned with the interplay between quasiconformal mappings and the space of exponentially integrable functions. Let us recall that a homeomorphism $f: \Omega \rightarrow \mathbb{R}^{n}$ defined on an open subset $\Omega$ of $\mathbb{R}^{n}$ (with $n \geq 2$ ) is a $K$ quasiconformal mapping if $f \in W_{\mathrm{loc}}^{1, n}\left(\Omega, \mathbb{R}^{n}\right)$ and

$$
|D f(x)|^{n} \leq K J_{f}(x) \quad \text { for a.e. } x \in \Omega,
$$

for some constant $K \geq 1$. Here $D f(x)$ stands for the differential matrix of $f$ and $J_{f}(x)=\operatorname{det} D f(x)$ denotes the Jacobian determinant of $f$. The norm $|D f(x)|$ of $D f(x)$ in (1) is defined as $|D f(x)|=\sup \left\{|D f(x) \xi|: \xi \in \mathbb{R}^{n},|\xi|=1\right\}$.

If $\Omega$ is a bounded domain of $\mathbb{R}^{n}$ with measure $|\Omega|$, the space of exponentially integrable functions $\operatorname{EXP}(\Omega)$ is the set of measurable functions $u: \Omega \rightarrow \mathbb{R}$ such that there exists $\lambda>0$ for which

$$
f_{\Omega} \exp \frac{|u(x)|}{\lambda} d x<\infty
$$

where the mean value notation $f_{\Omega}=(1 /|\Omega|) \int_{\Omega}$ is used. One of the interesting properties of functions in $\operatorname{EXP}(\Omega)$ consists in the fact that they may be characterized as BMO-majorized functions. Indeed, in [1] it is proved that $u \in \operatorname{EXP}(\Omega)$ if and only if there exists $v \in \operatorname{BMO}\left(\mathbb{R}^{n}\right)$ such that

$$
|u(x)| \leq v(x) \quad \text { a.e. } x \in \Omega \text {. }
$$

For the definition of the space $\operatorname{BMO}\left(\mathbb{R}^{n}\right)$ of functions of bounded mean oscillation see Section 2 below.

Quasiconformal mappings $f: \mathbb{R}^{n} \rightarrow \mathbb{R}^{n}$ and BMOfunctions $u: \mathbb{R}^{n} \rightarrow \mathbb{R}$ are related by the fact that the composition operator $u \mapsto u \circ f^{-1}$ maps $\mathrm{BMO}\left(\mathbb{R}^{n}\right)$ into itself continuously, as stated by a result of Reimann [2]: there exists $C=C(n, K) \geq 1$ such that, for every $u \in \operatorname{BMO}\left(\mathbb{R}^{n}\right)$, one has

$$
\begin{aligned}
\frac{1}{C(n, K)}\|u\|_{\mathrm{BMO}\left(\mathbb{R}^{n}\right)} & \leq\left\|u \circ f^{-1}\right\|_{\mathrm{BMO}\left(\mathbb{R}^{n}\right)} \\
& \leq C(n, K)\|u\|_{\mathrm{BMO}\left(\mathbb{R}^{n}\right)} .
\end{aligned}
$$

In light of the connection between exponentially integrable functions and functions of bounded mean oscillation, composition operators acting continuously on EXP have been considered in $[3,4]$, where it is proved that, given a $K$-quasiconformal mapping $f: \Omega \rightarrow \mathbb{R}^{n}$, there exists 
$C=C(n, K) \geq 1$ such that, for every $u \in \operatorname{EXP}(\Omega)$ and for every ball $B \subset \subset \Omega$, one has

$$
\begin{aligned}
\frac{1}{C(n, K)}\|u\|_{\operatorname{EXP}(B)} & \leq\left\|u \circ f^{-1}\right\|_{\operatorname{EXP}(f(B))} \\
& \leq C(n, K)\|u\|_{\operatorname{EXP}(B)} .
\end{aligned}
$$

The estimates above may be seen as the analogy of (4) in the framework of the space of exponentially integrable functions.

It is worth pointing out that spaces of functions of bounded mean oscillation and exponentially integrable functions are not the only ones which are stable under quasiconformal changes of variables. We recall that quasiconformal mappings and their generalizations provided by homeomorphisms of finite distortion or bi-Sobolev mappings (see, e.g., $[5,6])$, turn to be the class of homeomorphisms for which the composition operator acts continuously between Sobolev, Lorentz-Sobolev, and Zygmung-Sobolev spaces (see [7-9] and the references therein).

Explicit estimates of the constants appearing in (4) and (5) have been considered a problem of its own interest (see, e.g., [10] for an application). In the planar case, sharp estimates for the constant $C(2, K)$ appearing in (5) are given in [4]. As a refinement of the result of Reimann, explicit estimates of the constant $C(n, K)$ appearing in (4) are provided in [11]. More precisely, a constant $\widetilde{G}_{1}\left(J_{f}\right)$ depending on the Jacobian of $f$ may be defined (see Section 2.4 below) in such a way that the results of [11] may be stated as follows

$$
\left\|u \circ f^{-1}\right\|_{\mathrm{BMO}\left(\mathbb{R}^{n}\right)} \leq C(n) \widetilde{G}_{1}\left(J_{f}\right)\|u\|_{\mathrm{BMO}\left(\mathbb{R}^{n}\right)},
$$

for every $u \in \operatorname{BMO}\left(\mathbb{R}^{n}\right)$ and for some suitable constant $C=C(n) \geq 1$ depending only on the dimension $n$. The definition of the constant $\widetilde{G}_{1}\left(J_{f}\right)$ strongly relies on the fact that the Jacobian of a quasiconformal mapping is a weight in $A_{\infty}$ or equivalently in $G_{1}$ (see Section 2.4 for the definitions of such classes of weights). In particular, it is well known (see [12]) that $v \in G_{1}$ if and only if for every ball $B \subset \mathbb{R}^{n}$ and every measurable set $F \subset B$ it holds

$$
\frac{\int_{F} v(x) d x}{\int_{B} v(x) d x} \leq L\left(\frac{|F|}{|B|}\right)^{\beta},
$$

for some $0<\beta \leq 1 \leq L$ independent of $F$ and $B$. Equivalently (see again [12]) a weight $w \in A_{\infty}$ if and only if for every ball $B \subset \mathbb{R}^{n}$ and every measurable set $E \subset B$ it holds

$$
\frac{|E|}{|B|} \leq M\left(\frac{\int_{E} w(x) d x}{\int_{B} w(x) d x}\right)^{\alpha},
$$

for some $0<\alpha \leq 1 \leq M$ independent of $E$ and $B$.

The goal of this paper is to seek a precise estimate as in (6) in the framework of the class of exponentially integrable functions. To this aim, we define for a weight $v$ in $G_{1}$ the constant $\widehat{G}_{1}(v)$ as

$$
\begin{aligned}
& \widehat{G}_{1}(v)=\inf \left\{\max \left\{\frac{1}{\beta}, 1+\frac{\log L}{\beta}\right\}: 0<\beta \leq 1\right. \\
& \leq L \text { and (7) holds }\},
\end{aligned}
$$

and similarly, we define for a weight $w$ in $A_{\infty}$ the constant $\widehat{A}_{\infty}(w)$ as

$$
\begin{aligned}
& \widehat{A}_{\infty}(w)=\inf \left\{\max \left\{\frac{1}{\alpha}, 1+\frac{\log M}{\alpha}\right\}: 0<\alpha \leq 1\right. \\
& \leq M \text { and (8) holds }\} .
\end{aligned}
$$

Our main result reads as follows.

Theorem 1. Let $\Omega, \Omega^{\prime}$ be bounded domains of $\mathbb{R}^{n}, n \geq 2$. Let $f: \Omega \rightarrow \Omega^{\prime}$ be a $K$-quasiconformal mapping. Then

$$
\begin{aligned}
\frac{1}{\widehat{A}_{\infty}\left(J_{f}\right)}\|u\|_{\operatorname{EXP}(B)} & \leq\left\|u \circ f^{-1}\right\|_{\operatorname{EXP}(f(B))} \\
& \leq \widehat{G}_{1}\left(J_{f}\right)\|u\|_{\operatorname{EXP}(B)},
\end{aligned}
$$

for each ball $B \subset \subset \Omega$ and for every $u \in \operatorname{EXP}(\Omega)$.

The norm $\|\cdot\|_{\operatorname{EXP}(B)}$ is defined in (16) of Section 2.2 below.

Our result is sharp, in the sense that equalities are attained in (11) for special choices of the mapping $f$ (e.g., when $f$ is the identity map). We are able to obtain such optimal result since we give a characterization of constant weights in terms of the constants in (9) and (10) in Section 4. In particular, a $G_{1}$ weight $v$ is constant a.e. in $\mathbb{R}^{n}$ if and only if $\widehat{G}_{1}(v)=1$ and $\widehat{A}_{\infty}(v)=1$. Moreover, the result of Theorem 1 extends the one of [3], where the case of planar principal quasiconformal mappings has been considered. We call principal any quasiconformal mapping $f: \mathbb{R}^{2} \rightarrow \mathbb{R}^{2}$ which is conformal outside the unit disk $\mathbb{D}=\left\{x \in \mathbb{R}^{2}:|x|<\right.$ $1\}$ and which satisfies the following normalization

$$
|f(x)-x|=\mathcal{O}\left(\frac{1}{|x|}\right) \quad \text { if }|x|>1
$$

For this peculiar class of quasiconformal mappings, the following result has been previously established.

Theorem 2 (see [3]). Let $f: \mathbb{R}^{2} \rightarrow \mathbb{R}^{2}$ be a K-quasiconformal principal mapping which maps $\mathbb{D}$ onto itself. Then, the following estimates hold true

$$
\begin{aligned}
& \frac{1}{1+K \log K}\|u\|_{\operatorname{EXP}(\mathbb{D})} \leq\left\|u \circ f^{-1}\right\|_{\operatorname{EXP}(\mathbb{D})} \\
& \leq(1+K \log K)\|u\|_{\operatorname{EXP}(\mathbb{D})},
\end{aligned}
$$

\section{for every $u \in \operatorname{EXP}(\mathbb{D})$.}

We will provide an alternative proof of the previous result, which is based on the fact that estimate (11) reduces to (13) for a principal quasiconformal mapping which maps the unit disk onto itself. In general, a principal quasiconformal map in the plane does not necessarily send the unit disk onto itself. However, there exist nontrivial examples of principal 
quasiconformal mappings sending the unit disk onto itself, such as the radial stretching $f: \mathbb{R}^{2} \rightarrow \mathbb{R}^{2}$ of the form

$$
\begin{aligned}
& f(x)=|x|^{K} \frac{x}{|x|} \quad \text { for } x \in \mathbb{D}, \\
& f(x)=x \quad \text { for } x \in \mathbb{R}^{2} \backslash \mathbb{D} .
\end{aligned}
$$

The paper is organized as follows. Section 2 is devoted to the definitions of the function spaces object of our studies. In particular, the connection between BMO-functions and $A_{p^{-}}$ weights is treated. In Section 3 we give the proof of Theorem 1. In Section 4 we give the aforementioned characterization of the constant weights, which allows us to conclude the sharpness of Theorem 1. Finally, in Section 5 applications of Theorem 1 are given; in particular we provide a precise estimate which relates $\left\|\log J_{f^{-1}}\right\|_{\operatorname{EXP}(f(B))}$ and $\left\|\log J_{f}\right\|_{\operatorname{EXP}(B)}$ for any ball $B \subset \subset \Omega$.

\section{Preliminaries}

2.1. Properties of Quasiconformal Mappings. We report here some well-known facts about quasiconformal mappings. We recall that the change of variables formula holds for a quasiconformal mapping $f: \Omega \rightarrow \Omega^{\prime}$. More precisely, if $\varphi \in L_{\mathrm{loc}}^{1}\left(\Omega^{\prime}\right)$ then $(\varphi \circ f) J_{f} \in L_{\mathrm{loc}}^{1}(\Omega)$ and

$$
\int_{E} \varphi(f(x)) J_{f}(x) d x=\int_{f(E)} \varphi(y) d y,
$$

for every measurable $E \subset \subset \Omega$ (see, e.g., [13]). More generally, for an arbitrary Sobolev homeomorphism, the validity of the change of variables formula depends on the set of the points where the homeomorphism $f$ is approximately differentiable (see, e.g., [14]); more generally the condition of being absolutely continuous on lines plays an important role, especially for planar mappings (see, e.g., [15]), since very often properties of this type of mappings are sufficient to prove statements which one would assign to general Sobolev homeomorphisms.

2.2. Exponentially Integrable Functions. We recall (see, e.g., [16]) that $\operatorname{EXP}(\Omega)$ is a Banach space equipped with the norm

$$
\|u\|_{\operatorname{EXP}(\Omega)}=\sup _{0<t<|\Omega|} \frac{u^{*}(t)}{(1+\log (|\Omega| / t))},
$$

where $u^{*}$ is the nonincreasing rearrangement of $u$

$$
\begin{aligned}
u^{*}(t)=\sup \left\{\tau \geq 0: \mu_{u}(\tau)>t\right\} & \\
& \text { for every } t \in(0,|\Omega|),
\end{aligned}
$$

and $\mu_{u}$ is the distribution function of $u$

$$
\mu_{u}(\tau)=|\{x \in \Omega:|u(x)|>\tau\}| \text { for every } \tau \geq 0 .
$$

On the other hand, $\operatorname{EXP}(\Omega)$ may be also equipped with the Luxemburg norm defined as

$$
\||u|\|_{\operatorname{EXP}(\Omega)}=\inf \left\{\lambda>0: f_{\Omega} \exp \frac{|u(x)|}{\lambda} d x \leq 2\right\} .
$$

This norm is equivalent to the one in (16). As observed in [17], $L^{\infty}(\Omega)$ is not a dense subspace of $\operatorname{EXP}(\Omega)$. The distance to $L^{\infty}(\Omega)$ in the space $\operatorname{EXP}(\Omega)$ is defined as

$$
\operatorname{dist}_{\operatorname{EXP}(\Omega)}\left(u, L^{\infty}(\Omega)\right)=\inf _{\varphi \in L^{\infty}(\Omega)}\left|\|u-\varphi \mid\|_{\operatorname{EXP}(\Omega)} .\right.
$$

Appealing to the results in [17] the distance to $L^{\infty}(\Omega)$ in $\operatorname{EXP}(\Omega)$ evaluated with respect to the Luxemburg norm (19) is given by

$$
\begin{aligned}
& \operatorname{dist}_{\operatorname{EXP}(\Omega)}\left(u, L^{\infty}(\Omega)\right) \\
& \quad=\inf \left\{\lambda>0: f_{\Omega} \exp \frac{|u(x)|}{\lambda} d x<\infty\right\},
\end{aligned}
$$

for every $u \in \operatorname{EXP}(\Omega)$.

2.3. Functions of Bounded Mean Oscillation. A locally integrable function $u: \Omega \rightarrow \mathbb{R}$ has bounded mean oscillation, $u \in \operatorname{BMO}(\Omega)$, if

$$
\|u\|_{\mathrm{BMO}(\Omega)}=\sup _{B} f_{B}\left|u(x)-u_{B}\right| d x<\infty .
$$

The supremum in (22) is taken over all open balls $B \subset \Omega$ and the notation

$$
u_{B}=f_{B} u(x) d x,
$$

is used for averages.

2.4. $A_{p}$ and $G_{q}$ Classes. For our purposes, it is fundamental to introduce the Muckenhoupt class $A_{\infty}$ and the Gehring class $G_{1}$. First of all, we say that a measurable function $w: \mathbb{R}^{n} \rightarrow \mathbb{R}$ is a weight if $w$ is positive a.e. and locally integrable in $\mathbb{R}^{n}$. A weight $w$ belongs to the Muckenhoupt class $A_{\infty}$ if

$$
A_{\infty}(w)=\sup _{B}\left(f_{B} w d x\right)\left(\exp f_{B} \log \frac{1}{w} d x\right)<\infty .
$$

Similarly, a weight $v$ belongs to the Gehring class $G_{1}$ if

$$
G_{1}(v)=\sup _{B}\left(\exp f_{B} \frac{v}{v_{B}} \log \frac{v}{v_{B}} d x\right)<\infty .
$$

The suprema in (24) and (25) are taken over all balls $B \subset \mathbb{R}^{n}$.

The link between Muckenhoupt and Gehring classes is given in $[18,19]$ where it is proved that

$$
A_{\infty}=G_{1} .
$$

As a corollary of Gehring's Lemma [20], Jacobians of quasiconformal mappings are weights in the $A_{\infty}$ (or equivalently $G_{1}$ ) class. By virtue of the change of variables formula for quasiconformal mappings (see Section 2.1 above) and (7), for every ball $B \subset \mathbb{R}^{n}$ and every measurable set $F \subset B$ it holds

$$
\frac{|f(F)|}{|f(B)|} \leq L\left(\frac{|F|}{|B|}\right)^{\beta},
$$

for some $0<\beta \leq 1 \leq L$ independent of $F$ and $B$. 
For a weight $v$ in $G_{1}$, in [21] (and also studied in [22]) the constant $\widetilde{G}_{1}(v)$ is defined as

$$
\widetilde{G}_{1}(v)=\inf \left\{\frac{L}{\beta}: 0<\beta \leq 1 \leq L \text { and (7) holds }\right\} .
$$

We briefly refer to $\widetilde{G}_{1}(v)$ as the $\widetilde{G}_{1}$-constant of $v$.

As for (27), the change of variables formula for quasiconformal mappings and (8) immediately give us, for every ball $B \subset \mathbb{R}^{n}$ and every measurable set $E \subset B$

$$
\frac{|E|}{|B|} \leq M\left(\frac{|f(E)|}{|f(B)|}\right)^{\alpha}
$$

for some $0<\alpha \leq 1 \leq M$ independent of $E$ and $B$.

As done for the $\widetilde{G}_{1}$-constant, in [21] a second auxiliary constant is defined as

$$
\begin{aligned}
& \widetilde{A}_{\infty}(w) \\
& \quad=\inf \left\{\frac{M}{\alpha}: 0<\alpha \leq 1 \leq M \text { and (8) holds }\right\} .
\end{aligned}
$$

We briefly refer to $\widetilde{A}_{\infty}(w)$ as the $\widetilde{A}_{\infty}$-constant of $w$.

It is worth pointing out that to each function $u \in$ $\operatorname{BMO}\left(\mathbb{R}^{n}\right)$ there corresponds a weight in the $G_{1}$ class of Gehring given by $e^{\delta u}$, for some $\delta>0$ depending on $n$ and $\|u\|_{\text {BMO }}$ (see [23]). Conversely (see again [23]) if $v \in G_{1}$ (or equivalently if $v \in A_{\infty}$ ) then $\log v \in \mathrm{BMO}\left(\mathbb{R}^{n}\right)$. In particular, $\log J_{f}$ is a BMO-function, whenever $f: \mathbb{R}^{n} \rightarrow \mathbb{R}^{n}$ is a quasiconformal mapping.

For the sake of completeness, we also recall the definition of the Muckenhoupt class $A_{p}$ for $1 \leq p<\infty$. A weight $w$ belongs to the Muckenhoupt class $A_{p}$ for $1<p<\infty$ if

$$
A_{p}(w)=\sup _{B}\left(f_{B} w d x\right)\left(f_{B} w^{-1 /(p-1)} d x\right)^{p-1}<\infty .
$$

As a natural extension of the above definition, one can consider the Muckenhoupt class $A_{1}$ which covers the limit case $p=1$. A weight $w$ belongs to the Muckenhoupt class $A_{1}$ if

$$
A_{1}(w)=\sup _{B} \frac{f_{B} w d x}{\operatorname{ess} \inf _{x \in B} w(x)}<\infty .
$$

The suprema in (31) and (32) are taken over all balls $B \subset \mathbb{R}^{n}$. For each $1 \leq p<\infty$ we call $A_{p}(w)$ the $A_{p}$-constant of the weight $w$.

We recall here the definition of the Gehring class $G_{q}$ for $1<q \leq \infty$. A weight $v$ belongs to the Gehring class $G_{q}$ for $1<q<\infty$ if

$$
G_{q}(v)=\sup _{B}\left[\frac{\left(f_{B} v^{q} d x\right)^{1 / q}}{f_{B} v d x}\right]^{q /(q-1)}<\infty .
$$

As a natural extension of the above definition, one can consider the class $G_{\infty}$ which cover the limit case $q=\infty$. A weight $v$ belongs to the Gehring class $G_{\infty}$ if

$$
G_{\infty}(v)=\sup _{B} \frac{\operatorname{ess} \sup _{x \in B} v(x)}{f_{B} v d x}<\infty
$$

The suprema in (33) and (34) are taken over all balls $B \subset \mathbb{R}^{n}$. For each $1<q \leq \infty$ we call $G_{q}(v)$ the $G_{q}$-constant of the weight $v$. Each weight in the $G_{q}$ class satisfies a reverse Hölder inequality. This is a key fact in order to study the regularity of the Jacobian of quasiconformal mappings (see [20]).

For more details related to the Muckenhoupt and Gehring classes we refer to $[19,23,24]$.

\section{Explicit Bounds}

We start by proving Theorem 1 .

Proof of Theorem 1. Let us assume that $L$ and $\beta$ are arbitrary constants for which (27) holds, with $0<\beta \leq 1 \leq L$. We notice that for every $t>0$

$$
\begin{aligned}
\{y & \left.\in f(B):\left|u\left(f^{-1}(y)\right)\right|>t\right\} \\
& =f(\{x \in B:|u(x)|>t\}) .
\end{aligned}
$$

We compare the distribution functions of $u$ and $u \circ f^{-1}$ by means of the estimate (27) and we obtain

$$
\begin{aligned}
\mu_{u \circ f^{-1}}(t) & =\left|\left\{y \in f(B):\left|u\left(f^{-1}(y)\right)\right|>t\right\}\right| \\
& =|f(\{x \in B:|u(x)|>t\})| \\
& \leq L|f(B)|\left(\frac{\mu_{u}(t)}{|B|}\right)^{\beta} .
\end{aligned}
$$

Let $s \in(0,|f(B)|)$ and let $t \geq 0$ be such that

$$
\mu_{u \circ f^{-1}}(t)>s .
$$

From (36) we get

$$
\mu_{u}(t)>|B|\left(\frac{s}{L|f(B)|}\right)^{1 / \beta},
$$

and therefore, from the definition of nonincreasing rearrangement (17), we obtain that

$$
\left(u \circ f^{-1}\right)^{*}(s) \leq u^{*}\left(|B|\left(\frac{s}{L|f(B)|}\right)^{1 / \beta}\right) .
$$

We deduce directly from the definition of the norm (16) that

$$
\begin{aligned}
& u^{*}\left(|B|\left(\frac{s}{L|f(B)|}\right)^{1 / \beta}\right) \\
& \quad \leq\|u\|_{\operatorname{EXP}(B)}\left\{1+\frac{1}{\beta}\left[\log \frac{|f(B)|}{s}+\log L\right]\right\} .
\end{aligned}
$$

Combining (39) and (40) we obtain

$$
\begin{aligned}
& \left(u \circ f^{-1}\right)^{*}(s) \\
& \quad \leq\|u\|_{\operatorname{EXP}(B)}\left\{1+\frac{1}{\beta}\left[\log \frac{|f(B)|}{s}+\log L\right]\right\} .
\end{aligned}
$$


Let us introduce the function

$$
\phi_{L, \beta}(s)=1+\frac{1}{\beta}\left[\log \frac{|f(B)|}{s}+\log L\right]
$$

so that (41) may be rewritten as

$$
\left(u \circ f^{-1}\right)^{*}(s) \leq\|u\|_{\operatorname{EXP}(B)} \phi_{L, \beta}(s),
$$

and let us define

$$
\begin{aligned}
\psi_{L, \beta}(s) & =\frac{\phi_{L, \beta}(s)}{1+\log (|f(B)| / s)} \\
& =\frac{1+(1 / \beta)[\log (|f(B)| / s)+\log L]}{1+\log (|f(B)| / s)} .
\end{aligned}
$$

Our aim is to show that $\psi_{L, \beta}$ is bounded in $(0,|f(B)|)$ and we want to compute explicitly its upper bound. To this aim, we compute the derivative of $\psi_{L, \beta}$

$$
\frac{d \psi_{L, \beta}}{d s}(s)=-\frac{1-\beta-\log L}{\beta s(1+\log (|f(B)| / s))^{2}}
$$

so, the monotonicity of the function $\psi_{L, \beta}$ depends only on the sign of the constant $M(L, \beta):=1-\beta-\log L$ and for every $s \in(0,|f(B)|)$ we deduce

$$
\begin{aligned}
& \psi_{L, \beta}(s) \leq \frac{1}{\beta} \quad \text { if } 1-\beta-\log L \geq 0 \\
& \psi_{L, \beta}(s) \leq 1+\frac{\log L}{\beta} \quad \text { if } 1-\beta-\log L \leq 0 .
\end{aligned}
$$

The definition of $\psi_{L, \beta}$ (see (44)) and (46) imply

$$
\phi_{L, \beta}(s) \leq \max \left\{\frac{1}{\beta}, 1+\frac{\log L}{\beta}\right\}\left(1+\log \frac{|f(B)|}{s}\right) .
$$

Combining the latter estimate with (43) we conclude that

$$
\begin{aligned}
& \left(u \circ f^{-1}\right)^{*}(s) \\
& \quad \leq \max \left\{\frac{1}{\beta}, 1+\frac{\log L}{\beta}\right\}\|u\|_{\operatorname{EXP}(B)}\left(1+\log \frac{|f(B)|}{s}\right)
\end{aligned}
$$

and, by virtue of the definition of the norm in (16), we conclude that

$$
\left\|u \circ f^{-1}\right\|_{\operatorname{EXP}(f(B))} \leq \max \left\{\frac{1}{\beta}, 1+\frac{\log L}{\beta}\right\}\|u\|_{\operatorname{EXP}(B)} .
$$

Due to the definition of the constant $\widehat{G}_{1}\left(J_{f}\right)$ and to the fact that $L$ and $\beta$ are arbitrary constants for which (27) holds, we obtain

$$
\left\|u \circ f^{-1}\right\|_{\operatorname{EXP}(f(B))} \leq \widehat{G}_{1}\left(J_{f}\right)\|u\|_{\operatorname{EXP}(B)}
$$

for each ball $B \subset \subset \Omega$ and for every $u \in \operatorname{EXP}(\Omega)$.
It remains to prove the first inequality in (11). Let us assume that $M$ and $\alpha$ are arbitrary constants for which (29) holds, with $0<\alpha \leq 1 \leq M$. As before, we compare the distribution functions of $u$ and $u \circ f^{-1}$. This time we make use of the estimate (29) instead of (27) and we argue as in the proof of estimate (36). Indeed, if we pick $E=\{x \in B$ : $|u(x)|>t\}$ we obtain

$$
\begin{aligned}
\mu_{u}(t) & =|\{x \in B:|u(x)|>t\}| \\
& \leq M|B|\left(\frac{|f(\{x \in B:|u(x)|>t\})|}{|f(B)|}\right)^{\alpha} \\
& =M|B|\left(\frac{\mu_{u^{\circ} f^{-1}}(t)}{|f(B)|}\right)^{\alpha} .
\end{aligned}
$$

Let $s \in(0,|B|)$ and let $t \geq 0$ be such that

$$
\mu_{u}(t)>s
$$

From (51) we get

$$
s<M|B|\left(\frac{\mu_{u \circ f^{-1}}(t)}{|f(B)|}\right)^{\alpha}
$$

which in turn implies

$$
\mu_{u \circ f^{-1}}(t)>|f(B)|\left(\frac{s}{M|B|}\right)^{1 / \alpha} .
$$

It follows from the definition of nonincreasing rearrangement (17) that

$$
u^{*}(s) \leq\left(u \circ f^{-1}\right)^{*}\left(|f(B)|\left(\frac{s}{M|B|}\right)^{1 / \alpha}\right) .
$$

The argument which leads to (41) and the definition of the norm in (16), allows us to conclude that

$$
\begin{aligned}
& u^{*}(s) \\
& \quad \leq\left\|u \circ f^{-1}\right\|_{\operatorname{EXP}(f(B))}\left\{1+\frac{1}{\alpha}\left[\log \frac{|B|}{s}+\log M\right]\right\} .
\end{aligned}
$$

Arguing as in the proof of estimate (49) we have

$$
\|u\|_{\operatorname{EXP}(B)} \leq \max \left\{\frac{1}{\alpha}, 1+\frac{\log M}{\alpha}\right\}\left\|u \circ f^{-1}\right\|_{\operatorname{EXP}(f(B))} .
$$

Due to the definition of the constant $\widehat{A}_{\infty}\left(J_{f}\right)$ and to the fact that $M$ and $\alpha$ are arbitrary constants for which (29) holds, we obtain

$$
\|u\|_{\operatorname{EXP}(B)} \leq \widehat{A}_{\infty}\left(J_{f}\right)\left\|u \circ f^{-1}\right\|_{\operatorname{EXP}(f(B))},
$$

for each ball $B \subset \subset \Omega$ and for every $u \in \operatorname{EXP}(\Omega)$. The proof is complete.

We are in a position to prove Theorem 2. 
Proof of Theorem 2. Any principal quasiconformal mapping in the plane, which maps the unit disk onto itself, satisfies

$$
\frac{|f(E)|}{|f(\mathbb{D})|} \leq K\left(\frac{|E|}{|\mathbb{D}|}\right)^{1 / K},
$$

for every measurable subset $E \subset \mathbb{D}$ (see [25, Theorem 13.1.4]). Appealing to the definition (9) we deduce that

$$
\begin{aligned}
& \widehat{G}_{1}\left(J_{f}\right)=\inf \left\{\max \left\{\frac{1}{\beta}, 1+\frac{\log L}{\beta}\right\}: 0<\beta \leq 1\right. \\
& \leq L \text { and (27) holds }\},
\end{aligned}
$$

therefore

$$
\widehat{G}_{1}\left(J_{f}\right) \leq \max \{K, 1+K \log K\},
$$

directly follows from (59).

We introduce the auxiliary function

$$
\psi(K)=1+K \log K-K .
$$

Since $K \geq 1$ we have $\psi^{\prime}(K)=\log K \geq 0$ and in particular $\psi(K) \geq \psi(1)=0$, that is

$$
\max \{K, 1+K \log K\}=1+K \log K .
$$

Combining the latter identity with (61) we conclude that

$$
\widehat{G}_{1}\left(J_{f}\right) \leq 1+K \log K \text {. }
$$

The proof is complete, since (11) infers (13) in case of any $K$ quasiconformal principal mapping $f: \mathbb{R}^{2} \rightarrow \mathbb{R}^{2}$ which maps DD onto itself.

\section{A Characterization of Constant Weights}

We explicitly remark that, for a weight $v$ in $A_{\infty}$, one always has

$$
\widetilde{A}_{\infty}(v)=1 \quad \text { iff } v \text { is constant a.e. in } \mathbb{R}^{n} \text {. }
$$

This result is proved in [21]. With a similar proof, one can show the same property for the $\widetilde{G}_{1}$-constant, that is

$$
\widetilde{G}_{1}(v)=1 \text { iff } v \text { is constant a.e. in } \mathbb{R}^{n} .
$$

Our next result gives a similar characterization of constant weights in terms of the constant appearing in (9) and (10). It is crucial to prove sharpness of Theorem 1.

Proposition 3. Let $w$ be an $A_{\infty}$ weight such that $\widehat{A}_{\infty}(w)=1$. Then $w$ is constant a.e. in $\mathbb{R}^{n}$.

Proof of Proposition 3. Let us fix some integer $j \geq 1$. By the definition (10) of $\widehat{A}_{\infty}(w)$, we may find two sequences $\left\{\alpha_{j}\right\}$ and $\left\{M_{j}\right\}$ fulfilling

$$
\begin{aligned}
& 0<\alpha_{j} \leq 1 \leq M_{j}, \\
& 1 \leq \max \left\{\frac{1}{\alpha_{j}}, 1+\frac{\log M_{j}}{\alpha_{j}}\right\}<1+\frac{1}{j}
\end{aligned}
$$

and for every ball $B \subset \mathbb{R}^{n}$ and every measurable set $E \subset B$ it holds

$$
M_{j}\left(\frac{|E|}{|B|}\right)^{\alpha_{j}} \leq \frac{\int_{E} w(x) d x}{\int_{B} w(x) d x} .
$$

Moreover, $\alpha_{j}$ and $M_{j}$ are independent of $E$ and $B$. Because of condition (67), we may assume (up to a subsequence) that $\alpha_{j}$ converges to some $\alpha_{0} \in[0,1]$. Since one clearly has $1 / \alpha_{j} \geq 1$ for every $j \geq 1$, condition (67) implies $\alpha_{0}=1$. Conditions (67) and (68) also imply

$$
0 \leq \log M_{j}<\frac{\alpha_{j}}{j} .
$$

Appealing to the fact that $\alpha_{j} \rightarrow 1$ as $j \rightarrow \infty$, the latter relation implies $M_{j} \rightarrow 1$ as $j \rightarrow \infty$. Therefore, we may pass to the limit as $j \rightarrow \infty$ in (69) in order to get

$$
\frac{|E|}{|B|} \leq \frac{\int_{E} w(x) d x}{\int_{B} w(x) d x},
$$

for every ball $B \subset \mathbb{R}^{n}$ and every measurable set $E \subset B$. In particular, for every ball $B \subset \mathbb{R}^{n}$ and for a.e. $x_{0} \in B$ we have

$$
f_{B} w d x \leq w\left(x_{0}\right)
$$

which in turn implies that $w$ is a constant function.

Adapting suitably the proof of Proposition 3 one can prove next result featuring the $\widehat{G}_{1}(\cdot)$-constant.

Proposition 4. Let $v$ be an $G_{1}$ weight such that $\widehat{G}_{1}(v)=1$. Then $v$ is constant a.e. in $\mathbb{R}^{n}$.

\section{The Logarithm of the Jacobian of a Quasiconformal Mapping}

Let $f: \Omega \rightarrow \mathbb{R}^{n}$ be a quasiconformal mapping. In Section 2.4 we mentioned that $\log J_{f}$ is a BMO-function. Similarly (see, e.g., [4]) $\log J_{f} \in \operatorname{EXP}(\Omega)$. Our next results are consequences of Theorem 1 and relate the logarithm of the Jacobian of a quasiconformal mapping with the one of its inverse taking into account the constants $\widehat{G}_{1}\left(J_{f}\right)$ and $\widehat{A}_{\infty}\left(J_{f}\right)$. Moreover, these results can be seen as the counterpart in the space of exponentially integrable functions of the results of Reimann [10] in the setting of the BMO-space.

Corollary 5. Let $\Omega, \Omega^{\prime}$ be bounded domains of $\mathbb{R}^{n}, n \geq 2$. Let $f: \Omega \rightarrow \Omega^{\prime}$ be a K-quasiconformal mapping and let $\widehat{G}_{1}\left(J_{f}\right)$ be the constant defined in (9). Then

$$
\begin{aligned}
& \frac{1}{\widehat{A}_{\infty}\left(J_{f}\right)}\left\|\log J_{f}\right\|_{\operatorname{EXP}(B)} \leq\left\|\log J_{f^{-1}}\right\|_{\operatorname{EXP}(f(B))} \\
& \quad \leq \widehat{G}_{1}\left(J_{f}\right)\left\|\log J_{f}\right\|_{\operatorname{EXP}(B)}
\end{aligned}
$$

for each ball $B \subset \subset \Omega$ and for every $u \in \operatorname{EXP}(\Omega)$. 
Corollary 6. Let $f: \mathbb{R}^{n} \rightarrow \mathbb{R}^{n}$ be a quasiconformal mapping with $n \geq 2$. Then, there exists a constant $C=C(n) \geq 1$ depending only on $n$, such that

$$
\begin{aligned}
& \frac{1}{C(n) \widetilde{G}_{1}\left(J_{f^{-1}}\right)}\left\|\log J_{f}\right\|_{\mathrm{BMO}} \leq\left\|\log J_{f^{-1}}\right\|_{\mathrm{BMO}} \\
& \leq C(n) \widetilde{G}_{1}\left(J_{f}\right)\left\|\log J_{f}\right\|_{\mathrm{BMO}} .
\end{aligned}
$$

Proof of Corollary 5. We start by observing (see, e.g., [13]) that

$$
J_{f^{-1}}(f(x))=\frac{1}{J_{f}(x)} \quad \text { for a.e. } x \in \Omega
$$

Therefore, we may write the following identity for the Jacobian of $f$

$$
-\left(\log J_{f}\right) \circ f^{-1}=\log J_{f^{-1}}
$$

then, from (11), we have

$$
\left\|\log J_{f^{-1}}\right\|_{\operatorname{EXP}(f(B))} \leq \widehat{G}_{1}\left(J_{f}\right)\left\|\log J_{f}\right\|_{\operatorname{EXP}(B)} .
$$

It remains to prove the first inequality in (73). Consider the first inequality in (11), namely

$$
\|u\|_{\operatorname{EXP}(B)} \leq \widehat{A}_{\infty}\left(J_{f}\right)\left\|u \circ f^{-1}\right\|_{\operatorname{EXP}(f(B))},
$$

We recall that $f$ and $f^{-1}$ are both quasiconformal. So, we pick $u=v \circ f$ where $v$ is any function in $\operatorname{EXP}\left(\Omega^{\prime}\right)$, then (78) gives us

$$
\|v \circ f\|_{\operatorname{EXP}(B)} \leq \widehat{A}_{\infty}\left(J_{f}\right)\|v\|_{\operatorname{EXP}(f(B))} .
$$

In (76) we may replace $f$ by $f^{-1}$, so that

$$
-\log J_{f}=\left(\log J_{f^{-1}}\right) \circ f .
$$

Thus, (79) and (80) infer

$$
\left\|\log J_{f}\right\|_{\operatorname{EXP}(B)} \leq \widehat{A}_{\infty}\left(J_{f}\right)\left\|\log J_{f^{-1}}\right\|_{\operatorname{EXP}(f(B))} .
$$

The desired inequality (73) is proved.

Proof of Corollary 6. As in the proof of Corollary 5, we know that (76) holds. Then from (6), we have

$$
\left\|\log J_{f^{-1}}\right\|_{\mathrm{BMO}} \leq C(n) \widetilde{G}_{1}\left(J_{f}\right)\left\|\log J_{f}\right\|_{\mathrm{BMO}} .
$$

It remains to prove the first inequality in (74). In (82) we may replace $f$ by $f^{-1}$. Hence

$$
\left\|\log J_{f}\right\|_{\mathrm{BMO}} \leq C(n) \widetilde{G}_{1}\left(J_{f^{-1}}\right)\left\|\log J_{f^{-1}}\right\|_{\mathrm{BMO}} .
$$

The desired inequality (74) is proved.

Next results provide quantitative estimates as in (73) and (74) where the norms are replaced by the distances to $L^{\infty}$.
Corollary 7. Let $\Omega, \Omega^{\prime}$ be bounded domains of $\mathbb{R}^{n}, n \geq 2$. Let $f: \Omega \rightarrow \Omega^{\prime}$ be a K-quasiconformal mapping and let $\widehat{G}_{1}\left(J_{f}\right)$ be the constant defined in (9). Then

$$
\begin{aligned}
& \operatorname{dist}_{\mathrm{EXP}(f(B))}\left(\log J_{f^{-1}}, L^{\infty}(f(B))\right) \\
& \leq \widehat{G}_{1}\left(J_{f}\right) \operatorname{dist}_{\mathrm{EXP}(B)}\left(\log J_{f}, L^{\infty}(B)\right), \\
& \frac{1}{\widehat{A}_{\infty}\left(J_{f}\right)} \operatorname{dist}_{\mathrm{EXP}(B)}\left(\log J_{f}, L^{\infty}(B)\right) \\
& \quad \leq \operatorname{dist}_{\operatorname{EXP}(f(B))}\left(\log J_{f^{-1}}, L^{\infty}(f(B))\right)
\end{aligned}
$$

for each ball $B \subset \subset \Omega$.

Corollary 8. Let $f: \mathbb{R}^{n} \rightarrow \mathbb{R}^{n}$ be a quasiconformal mapping with $n \geq 2$. Then, there exists a constant $C=C(n)$ depending only on $n$, such that

$$
\begin{aligned}
& \operatorname{dist}_{\mathrm{BMO}}\left(\log J_{f^{-1}}, L^{\infty}\right) \\
& \leq C(n) \widetilde{G}_{1}\left(J_{f}\right) \operatorname{dist}_{\mathrm{BMO}}\left(\log J_{f}, L^{\infty}\right), \\
& \frac{1}{C(n) \widetilde{G}_{1}\left(J_{f^{-1}}\right)} \operatorname{dist}_{\mathrm{BMO}}\left(\log J_{f}, L^{\infty}\right) \\
& \leq \operatorname{dist}_{\mathrm{BMO}}\left(\log J_{f^{-1}}, L^{\infty}\right) .
\end{aligned}
$$

Proof of Corollary 7. Let $\varphi \in L^{\infty}(B)$ and let $\psi=-\varphi \circ f^{-1}$. Then, from (11), we have

$$
\begin{aligned}
& \left\|\log J_{f^{-1}}-\psi\right\|_{\operatorname{EXP}(f(B))}=\left\|\left(\log J_{f}-\varphi\right) \circ f^{-1}\right\|_{\operatorname{EXP}(f(B))} \\
& \leq \widehat{G}_{1}\left(J_{f}\right)\left\|\log J_{f}-\varphi\right\|_{\operatorname{EXP}(B)} .
\end{aligned}
$$

It is clear that there exists a measurable set $N_{0}$ of zero measure such that $\varphi$ is bounded in $B \backslash N_{0}$. Thus, $\psi$ is bounded in $f(B) \backslash f\left(N_{0}\right)$; on the other hand, one has $\left|f\left(N_{0}\right)\right|=0$, since $f$ satisfies the Lusin condition (see, e.g., [13]). We proved that $\psi \in L^{\infty}(f(B))$. Thus, from (88) we know that

$$
\begin{aligned}
\operatorname{dist}_{\operatorname{EXP}(f(B))}\left(\log J_{f^{-1}}, L^{\infty}\right) \\
\quad \leq \widehat{G}_{1}\left(J_{f}\right)\left\|\log J_{f} \varphi\right\|_{\operatorname{EXP}(B)} .
\end{aligned}
$$

Since $\varphi$ is an arbitrary function in $L^{\infty}(B)$, we conclude that

$$
\begin{aligned}
& \operatorname{dist}_{\operatorname{EXP}(f(B))}\left(\log J_{f^{-1}, L^{\infty}}\right) \\
& \quad \leq \widehat{G}_{1}\left(J_{f}\right) \operatorname{dist}_{\operatorname{EXP}(B)}\left(\log J_{f}, L^{\infty}\right) .
\end{aligned}
$$

Now we prove the inequality in (85). Thanks to (79) and arguing as before, we may conclude that

$$
\begin{aligned}
& \operatorname{dist}_{\operatorname{EXP}(B)}\left(v \circ f, L^{\infty}(B)\right) \\
& \quad \leq \widehat{A}_{\infty}\left(J_{f}\right) \operatorname{dist}_{\operatorname{EXP}(f(B))}\left(v, L^{\infty}(f(B))\right) .
\end{aligned}
$$


Thus

$$
\begin{aligned}
\text { dist }_{\mathrm{EXP}(B)}\left(\log J_{f}, L^{\infty}(B)\right) \\
\quad=\operatorname{dist}_{\mathrm{EXP}(B)}\left(\left(\log J_{f^{-1}}\right) \circ f, L^{\infty}(B)\right) \\
\quad \leq \widehat{A}_{\infty}\left(J_{f}\right) \operatorname{dist}_{\mathrm{EXP}(f(B))}\left(\log J_{f^{-1}}, L^{\infty}(f(B))\right) .
\end{aligned}
$$

The inequality in (85) is proved.

Proof of Corollary 8. Let $\varphi \in L^{\infty}\left(\mathbb{R}^{n}\right)$ and let $\psi=-\varphi \circ f^{-1}$. Then, from (6), we have

$$
\begin{aligned}
\left\|\log J_{f^{-1}}-\psi\right\|_{\mathrm{BMO}} & =\left\|\left(\log J_{f}-\varphi\right) \circ f^{-1}\right\|_{\mathrm{BMO}} \\
& \leq C(n) \widetilde{G}_{1}\left(J_{f}\right)\left\|\log J_{f}-\varphi\right\|_{\mathrm{BMO}} .
\end{aligned}
$$

It is clear that there exists a measurable set $N_{0}$ of zero measure such that $\varphi$ is bounded in $\mathbb{R}^{n} \backslash N_{0}$. Thus, $\psi$ is bounded in $\mathbb{R}^{n} \backslash f\left(N_{0}\right)$; on the other hand, one has $\left|f\left(N_{0}\right)\right|=0$, since $f$ satisfies the Lusin condition. We proved that $\psi \in L^{\infty}\left(\mathbb{R}^{n}\right)$. Thus, from (93) we know that

$$
\begin{aligned}
& \operatorname{dist}_{\mathrm{BMO}}\left(\log J_{f^{-1}}, L^{\infty}\right) \\
& \quad \leq C(n) \widetilde{G}_{1}\left(J_{f}\right)\left\|\log J_{f}-\varphi\right\|_{\mathrm{BMO}} .
\end{aligned}
$$

Since $\varphi$ is an arbitrary function in $L^{\infty}(B)$, we conclude that

$$
\begin{aligned}
& \operatorname{dist}_{\mathrm{BMO}}\left(\log J_{f^{-1}}, L^{\infty}\right) \\
& \quad \leq C(n) \widetilde{G}_{1}\left(J_{f}\right) \operatorname{dist}_{\mathrm{BMO}}\left(\log J_{f}, L^{\infty}\right) .
\end{aligned}
$$

The inequality (87) follows by replacing $f$ by $f^{-1}$ in (95), namely

$$
\begin{aligned}
& \operatorname{dist}_{\mathrm{BMO}}\left(\log J_{f}, L^{\infty}\right) \\
& \quad \leq C(n) \widetilde{G}_{1}\left(J_{f^{-1}}\right) \operatorname{dist}_{\mathrm{BMO}}\left(\log J_{f^{-1}}, L^{\infty}\right) .
\end{aligned}
$$

Given a function $u \in \operatorname{BMO}\left(\mathbb{R}^{n}\right)$, we consider the quantity

$$
\varepsilon(u)=\inf \left\{\lambda>0: \sup _{B} \int_{B} \exp \frac{\left|u-u_{B}\right|}{\lambda} d x<\infty\right\},
$$

introduced in [26] by Garnett and Jones. The supremum in (97) is taken over all balls $B \subset \mathbb{R}^{n}$. The main result of [26] states that $\varepsilon(\cdot)$ is equivalent to the distance to $L^{\infty}\left(\mathbb{R}^{n}\right)$ in the space $\operatorname{BMO}\left(\mathbb{R}^{n}\right)$ defined as

$$
\operatorname{dist}_{\mathrm{BMO}\left(\mathbb{R}^{n}\right)}\left(u, L^{\infty}\left(\mathbb{R}^{n}\right)\right)=\inf _{\varphi \in L^{\infty}(\Omega)}\|u-\varphi\|_{\mathrm{BMO}\left(\mathbb{R}^{n}\right)} .
$$

We also refer to $[17,27]$ for the problem of finding a formula for the distance to $L^{\infty}$ in grand Sobolev and grand Orlicz spaces. The next result is an immediate consequence of the previous result.
Corollary 9. Let $f: \mathbb{R}^{n} \rightarrow \mathbb{R}^{n}$ be a quasiconformal mapping with $n \geq 2$. Then, there exists a constant $C=C(n)$ depending only on $n$, such that

$$
\begin{gathered}
\frac{1}{C(n) \widetilde{G}_{1}\left(J_{f^{-1}}\right)} \varepsilon\left(\log J_{f}\right) \leq \varepsilon\left(\log J_{f^{-1}}\right) \\
\leq C(n) \widetilde{G}_{1}\left(J_{f}\right) \varepsilon\left(\log J_{f}\right) .
\end{gathered}
$$

\section{Competing Interests}

The authors declare that there is no conflict of interest regarding the publication of this paper.

\section{Acknowledgments}

Raffaella Giova has been supported by the Gruppo Nazionale per l'Analisi Matematica, la Probabilità e le loro Applicazioni (GNAMPA) of the Istituto Nazionale di Alta Matematica (INdAM) and by Project Legge 5/2007 Regione Campania "Spazi pesati e applicazioni al calcolo delle variazioni".

\section{References}

[1] T. Iwaniec and C. Sbordone, "Quasiharmonic fields," Annales de l'Institut Henri Poincaré. Analyse Non Linéaire, vol. 18, no. 5, pp. 519-572, 2001.

[2] H. M. Reimann, "Functions of bounded mean oscillation and quasiconformal mappings," Commentarii Mathematici Helvetici, vol. 49, pp. 260-276, 1974.

[3] F. Farroni and R. Giova, "Quasiconformal mappings and exponentially integrable functions," Studia Mathematica, vol. 203, no. 2, pp. 195-203, 2011.

[4] F. Farroni and R. Giova, "Quasiconformal mappings and sharp estimates for the distance to $L^{\infty}$ in some function spaces," Journal of Mathematical Analysis and Applications, vol. 395, no. 2, pp. 694-704, 2012.

[5] C. Capone, M. R. Formica, R. Giova, and R. Schiattarella, "On the regularity theory of bi-Sobolev mappings," Atti della Accademia Nazionale dei Lincei. Rendiconti Lincei. Matematica e Applicazioni, vol. 24, no. 4, pp. 527-548, 2013.

[6] S. Hencl, G. Moscariello, A. Passarelli di Napoli, and C. Sbordone, "Bi-Sobolev mappings and elliptic equations in the plane," Journal of Mathematical Analysis and Applications, vol. 355, no. 1, pp. 22-32, 2009.

[7] B. Bojarski and T. Iwaniec, "Analytical foundations of the theory of quasiconformal mappings in $R^{n}$," Annales Academiae Scientiarum Fennicae. Series A I. Mathematica, vol. 8, no. 2, pp. 257-324, 1983.

[8] F. Farroni, R. Giova, G. Moscariello, and R. Schiattarella, "Homeomorphisms of finite inner distortion: composition operators on Zygmund-Sobolev and Lorentz-Sobolev spaces," Mathematica Scandinavica, vol. 116, no. 1, pp. 34-52, 2015.

[9] S. Hencl and L. Kleprlik, "Composition of q-quasiconformal mappings and functions in Orlicz-Sobolev spaces," Illinois Journal of Mathematics, vol. 56, no. 3, pp. 931-955, 2012.

[10] H. M. Reimann, "On the parametric representation of quasiconformal mappings," in Proceedings of the Convegno sulle Transformazioni Quasiconformi e Questioni Connesse, vol. 18 of Symposia Mathematica, pp. 421-428, Academic Press, Rome, Italy, 1976. 
[11] Y. Gotoh, "On composition operators which preserve BMO," Pacific Journal of Mathematics, vol. 201, no. 2, pp. 289-307, 2001.

[12] R. R. Coifman and C. Fefferman, "Weighted norm inequalities for maximal functions and singular integrals," Studia Mathematica, vol. 51, pp. 241-250, 1974.

[13] S. Rickman, Quasiregular Mappings (Ergebnisse der Mathematik und ihrer Grenzgebiete. 3. Folge/A Series of Modern Surveys in Mathematics), vol. 26, Springer, Berlin, Germany, 1993.

[14] P. Hajlasz, "Change of variables formula under minimal assumptions," Colloquium Mathematicum, vol. 64, no. 1, pp. 93101, 1993.

[15] F. Farroni, R. Giova, and C. Sbordone, "Regularity points of ACL-homeomorphisms in the plane," Contemporary Mathematics, vol. 594, pp. 167-178, 2013.

[16] C. Bennett and R. Sharpley, Interpolation of Operators, vol. 129 of Pure and Applied Mathematics, Academic Press, Boston, Mass, USA, 1988.

[17] M. Carozza and C. Sbordone, "The distance to $L^{\infty}$ in some function spaces and applications," Differential and Integral Equations, vol. 10, no. 4, pp. 599-607, 1997.

[18] R. Fefferman, "A criterion for the absolute continuity of the harmonic measure associated with an elliptic operator," Journal of the American Mathematical Society, vol. 2, no. 1, pp. 127-135, 1989.

[19] G. Moscariello and C. Sbordone, " $A_{\infty}$ as a limit case of reverseHölder inequalities when the exponent tends to 1," Ricerche di Matematica, vol. 44, no. 1, pp. 131-144, 1995.

[20] F. W. Gehring, “The $L^{p}$-integrability of the partial derivatives of a quasiconformal mapping," Acta Mathematica, vol. 130, pp. 265-277, 1973.

[21] T. Radice, "New bounds for Aळo weights," Annales Academice Scientiarum Fennico: Mathematica, vol. 33, no. 1, pp. 111-119, 2008.

[22] F. Farroni and R. Giova, "Change of variables for $A_{\infty}$ weights by means of quasiconformal mappings: sharp results," Annales Academiae Scientiarum Fennicae: Mathematica, vol. 38, pp. 785796, 2013.

[23] J. García-Cuerva and J. L. Rubio de Francia, Weighted Norm Inequalities and Related Topics, Elsevier, Berlin, Germany, 1985.

[24] A. Fiorenza, B. Gupta, and P. Jain, "The maximal theorem for weighted grand Lebesgue spaces," Studia Mathematica, vol. 188, no. 2, pp. 123-133, 2008.

[25] K. Astala, T. Iwaniec, and G. Martin, Elliptic Partial Differential Equations and Quasiconformal Mappings in the Plane, vol. 48 of Princeton Mathematical Series, Princeton University, Princeton, NJ, USA, 2009.

[26] J. B. Garnett and P. W. Jones, "The distance in BMO to $L^{\infty}$," Annals of Mathematics: Second Series, vol. 108, no. 2, pp. 373393, 1978.

[27] F. Farroni and R. Giova, "The distance to $L^{\infty}$ in the grand Orlicz spaces," Journal of Function Spaces and Applications, vol. 2013, Article ID 658527, 7 pages, 2013. 


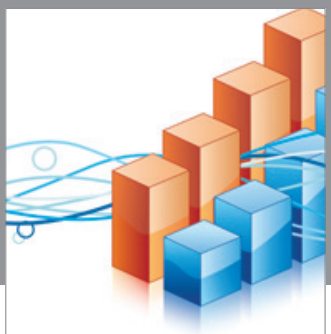

Advances in

Operations Research

vatem alat4

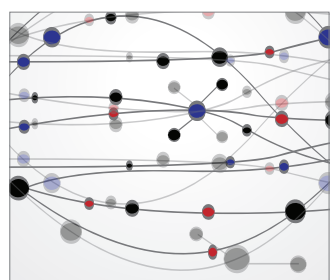

\section{The Scientific} World Journal
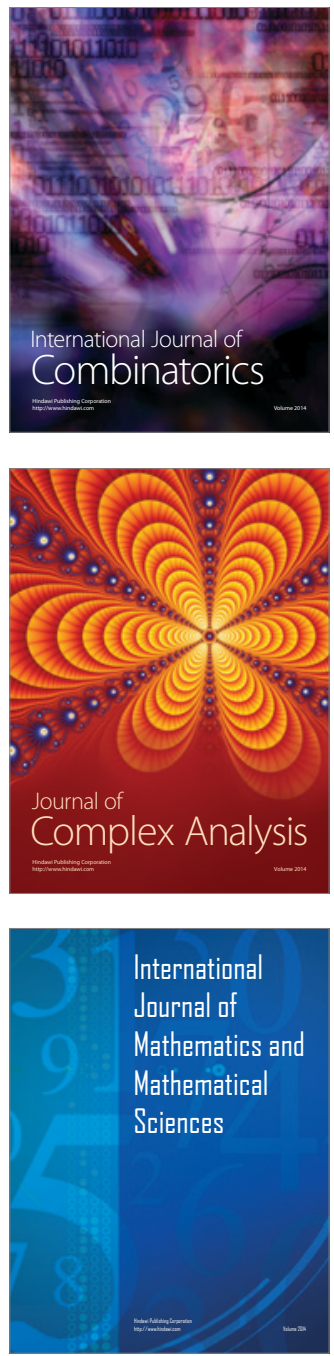
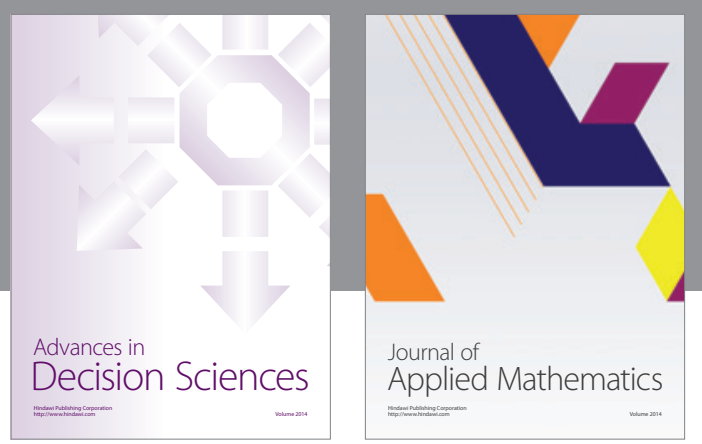

Algebra

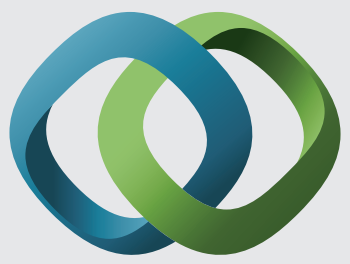

\section{Hindawi}

Submit your manuscripts at

http://www.hindawi.com
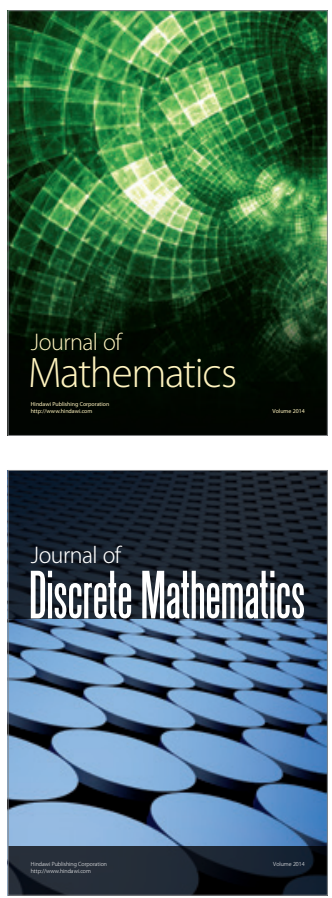

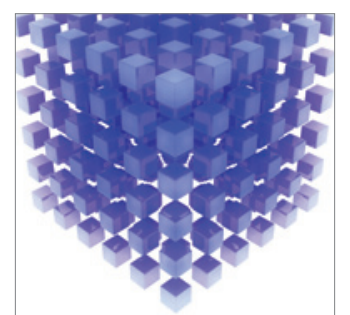

Mathematical Problems in Engineering
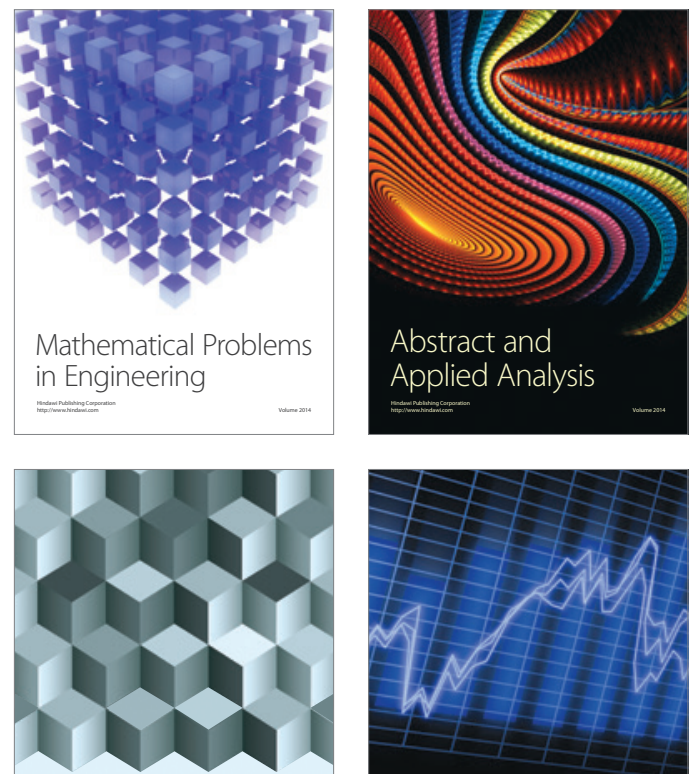

Journal of

Function Spaces

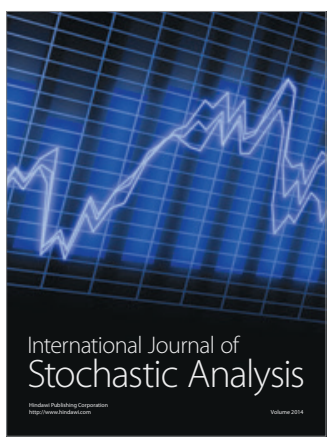

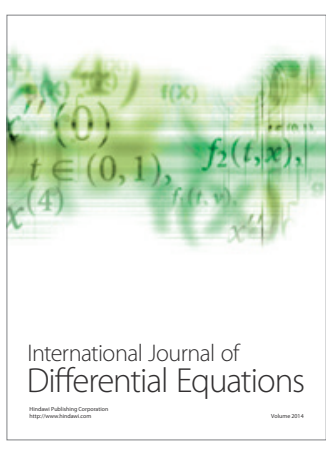
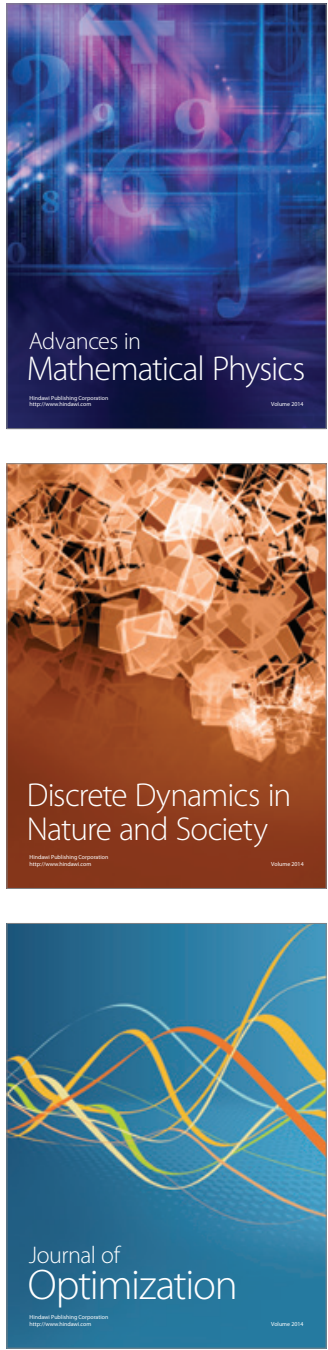